\title{
Estimating 24-h urinary sodium excretion from casual spot urine specimen among hypertensive patients in Northwest China: the Salt Substitute and Stroke Study
}

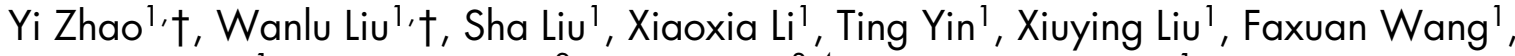 \\ Xiaoyu Chang ${ }^{1}$, Tianjing Zhang ${ }^{2}$, Maoyi Tian ${ }^{3,4}$ and Yuhong Zhang ${ }^{1, *}$ \\ 'Public Health and Management School of Ningxia Medical University, Yinchuan City, Ningxia, China: ${ }^{2} T$ he People's \\ Hospital of Anyang City, Anyang City, Henan Province, China: ${ }^{3}$ The George Institute for Global Health at Peking \\ University Health Science Center, Haidian District, Beijing 100088, China: ${ }^{4}$ The George Institute for Global Health, \\ University of New South Wales, Newtown, NSW 2042, Australia
}

Submitted 25 February 2019: Final revision received 12 November 2019: Accepted 3 December 2019: First published online 29 April 2020

\begin{abstract}
Objective: To develop an equation that can estimate the 24-h urinary Na excretion by using casual spot urine specimen for older hypertensive participants in rural Ningxia and further to compare with the INTERSALT method, Kawasaki method and Tanaka method.

Design: Older hypertensive participants in rural Ningxia provided their casual spot urine samples and 24-h urine samples between January 2015 and February 2017. Sex-specific equation was developed using linear forward stepwise regression analysis. Model fit was assessed using adjusted $R^{2}$. Approximately half of all participants were randomly selected to validate the equation. Mean differences, intraclass correlation coefficients and Bland-Altman plots were used to evaluate the performance of all methods.

Setting: Pingluo County and Qingtongxia County in Ningxia Hui Autonomous Region, China.

Participants: Older hypertensive participants in rural Ningxia.

Results: Totally, 807 of 1120 invited participants provided qualified 24-h urine samples and spot urine samples. There was no statistical difference comparing the laboratory-based method against the new method and the INTERSALT method, while Kawasaki method had the largest bias with a mean difference of $40 \cdot 81 \mathrm{~g} / \mathrm{d}$ (95\% CI $39.27,42.35 \mathrm{~g} / \mathrm{d}$ ). Bland-Altman plots showed similar pattern of the results.

Conclusion: The INTERSALT method and the new equation have the potential to estimate the 24-h urinary $\mathrm{Na}$ excretion in this study population. However, the extrapolation of the results to other population needs to be careful. Future research is required to establish a more reliable method to estimate 24-h urinary $\mathrm{Na}$ excretion.
\end{abstract}

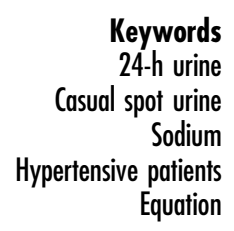

High $\mathrm{Na}$ intake is a modifiable risk factor for hypertension. Studies showed every $100 \mathrm{mmol} / \mathrm{d}$ of $\mathrm{Na}$ intake resulted in 5.8 and $3.8 \mathrm{mmHg}$ increases in systolic and diastolic blood pressure, respectively ${ }^{(1,2)}$. Excessive $\mathrm{Na}$ intake is also associated with cerebrovascular diseases and gastric cancer ${ }^{(3,4)}$. World Health Organization recommended the daily $\mathrm{Na}$ intake within $2 \mathrm{~g}$ (equivalent to $5 \mathrm{~g}$ of salt per d) for adults ${ }^{(5)}$. There is an urgent need to establish a feasible solution to monitor the $\mathrm{Na}$ intake on a population level. 24-h dietary

$†$ These authors contributed equally to this work. recall and 24-h urine collection remain as the two widely used methods to estimate population $\mathrm{Na}$ intake. 24-h dietary recall usually underestimated $\mathrm{Na}$ intake due to underreporting and intangible $\mathrm{Na}$ content in food ${ }^{(6)}$. 24-h urine collection is considered as the gold standard to assess dietary $\mathrm{Na}$ intake ${ }^{(7)}$, but it is expensive in large-scale population survey and burdensome to participants. Previous studies used casual spot urine specimen to replace 24-h urine to assess dietary $\mathrm{Na}$ intake ${ }^{(8-10)}$. INTERSALT method, Kawasaki method and Tanaka method have been established to estimate 24-h urinary $\mathrm{Na}$ excretion from spot urine ${ }^{(11-13)}$.

*Corresponding author: Email zhabour@163.com

(c) The Authors 2020. This is an Open Access article, distributed under the terms of the Creative Commons Attribution licence (http:// creativecommons.org/licenses/by/4.0/), which permits unrestricted re-use, distribution, and reproduction in any medium, provided the original work is properly cited. 
However, whether these three methods are valid to Chinese rural hypertensive patients remains unknown.

Therefore, the objectives of this study are to develop a method to estimate 24-h urinary $\mathrm{Na}$ excretion by using casual spot urine specimen in rural hypertensive patients in Ningxia Hui Autonomous Region (thereafter 'Ningxia') and further to compare this method with the other three established methods.

\section{Methods}

\section{Study sites and participants}

This study was a sub-study of the current ongoing largescale cluster randomised controlled trial: the Salt Substitute and Stroke Study. The trial was registered in clinicaltrial. gov (NCT02092090). A total of 120 villages from Pingluo County and Qingtongxia County in Ningxia participated in the Salt Substitute and Stroke Study trial. In each village, thirty-five individuals with high risk of stroke were recruited. The selection criteria of the participants included: (1) with a history of stroke; (2) or age $\geq 60$ years with uncontrolled high blood pressure (systolic blood pressure $\geq 140 \mathrm{mmHg}$ at visit if on blood pressure lowering medication or systolic blood pressure $\geq 160 \mathrm{mmHg}$ at visit if not on blood pressure lowering medication). A series of process indicators were measured in a random subset of individuals. Key indicators include blood pressure, medication use, urinary $\mathrm{Na}$ and urinary $\mathrm{K}$ measured from the $24 \mathrm{~h}$ urine and spot urine samples. The process indicators were collected from a random sample of twenty individuals drawn from a stratified random sample of at least six villages in each county (three from the intervention group and three from the control group). In each village, a randomised list of participants was generated. Interviewers invited the first twenty participants from the list to participate the survey. If anyone from the list was unable to take part in, the interviewer will move to the next available participant. Participants with the following conditions were excluded from the urine collection: (1) urinary incontinence; (2) unable to collect urine on its own and cannot find help from others; (3) acute or chronic urinary tract infection, vaginal infection and perianal infection; (4) acute or chronic urinary tract, vaginal and gastrointestinal bleeding; (5) females who were pregnant, breast-feeding or during menstruation (only allowed to participate after $2 \mathrm{~d}$ when their period was clear); (6) vomiting or diarrhoea. The process indicator survey was repeated every 12 months throughout the follow-up. Details of the Salt Substitute and Stroke Study trial were published elsewhere ${ }^{(14,15)}$.

\section{Specimen collection and analysis}

At each process indicator survey, participants were invited to collect both casual spot urine and 24-h urine samples. Participants were asked to provide a casual spot urine specimen (midstream urine) before the collection of $24-\mathrm{h}$ urine. The spot urine was collected into a disposable urine cup, and two $1.5 \mathrm{ml}$ aliquots were removed. 24-h urine collection started immediately after the spot urine collection completed. The participants were provided with six 1-litre plastic containers and instructed to collect all urine voided during a 24-h period according to standard procedures. Participants were asked to return all containers at the completion of the 24-h urine collection. Interviewers recorded the start and end time of 24-h urine collection, the total urine volume and participant's self-reported missed volume on a worksheet. 24-h urine specimens were excluded in any of the following conditions: (1) the first void of urine in the morning was missed; (2) the missed volume reported by the participant was over $10 \%$ of the total volume; (3) the urine was contaminated or (4) the participant reported vomiting or diarrhoea during the collection. In addition, samples were excluded from the analysis if 24 -h creatinine excretion was $<4 \mathrm{mmol}$ or $>25 \mathrm{mmol}$ in women or $<6 \mathrm{mmol}$ or $>30 \mathrm{mmol}$ in men.

The fieldwork was carried out between January 2015 and February 2017. Both spot urine and 24-h urine specimens were frozen at $-20^{\circ} \mathrm{C}$ within $4 \mathrm{~h}$ after collection. Specimens were then shipped to a central laboratory in Beijing for analysis. The concentration of urinary $\mathrm{Na}$ and $\mathrm{K}$ was analysed using ion selective electrode method, and urinary creatinine was analysed using sarcosine oxidase method with the HITACHI 7600 automated biochemistry analyzer. Weight, height and blood pressure were measured according to a standardised protocol. Participants' demographic information was also collected.

\section{Statistical analysis}

Continuous variables were described as mean $\pm \mathrm{SD}$, while categorical variable were described as proportions (\%). Spot urine $\mathrm{Na}$ concentration $\left(\mathrm{Na}_{\text {spot }}\right)$, spot urine $\mathrm{K}$ concentration $\left(\mathrm{K}_{\text {spot }}\right)$, spot urine creatinine concentration $\left(\mathrm{Cr}_{\text {spot }}\right)$, age, weight, height and BMI were used in the regression analysis to develop the new method. Age, weight, height and BMI are influence factors on volume of urinary, and creatinine concentration is thought to be constant throughout the day ${ }^{(16)}$. The factors mentioned above were included in published predictive equations: the INTERSALT equation, Kawasaki equation and Tanaka equation ${ }^{(11-13,17)}$. All participants' data were defined as the test data set to develop the sex-specific equations. Approximately $50 \%$ of all participants were randomly selected as validation data set to verify the equations. All regression analyses were linear forward stepwise regression analysis. Adjusted $R^{2}$ was used to assess the model fitting.

The performance of all methods was determined by comparing the laboratory-based method against the new method, INTERSALT method, Kawasaki method and Tanaka method. Mean difference, intraclass correlation coefficient and Bland-Altman plots were used to evaluate the agreement ${ }^{(18)}$. All statistical analyses were performed 
using SPSS (version 14.0, SPSS Corp.). Bland-Altman plots were drawn by Medcalc Software (version 15.2.2, MedCalc Software Crop.).

\section{Results}

\section{Study flow chart}

A total of 1120 individuals recruited between January 2015 and February 2017 were invited to participate in the survey. $162(14.46 \%)$ refused, whereas sixty-four (6.7\%) were not able to provide casual spot urine samples and 73 (7.6\%) provided unqualified 24 -h urine samples. In addition, 14 (1.5\%) individuals were excluded from data analysis due to the missing data. Finally, 807 (84.2\%) participants were included in the statistical analysis. Details are shown in Fig. 1.

\section{Selected characteristics of the participants}

In total, a typical participant was a 68.9 years old male. Selected characteristics of the participants are shown in Supplemental Table 1.

\section{Regression models}

$\mathrm{Na}_{\text {spot }}(\mathrm{mmol} / \mathrm{l}), \mathrm{K}_{\text {spot }}(\mathrm{mmol} / \mathrm{l}), \mathrm{Cr}_{\text {spot }}(\mathrm{mmol} / \mathrm{l})$, age, height (cm), weight $(\mathrm{kg})$ and BMI were used in the linear stepwise regression analysis and the gender-specific regression models. Included and removed variables were presented in Supplemental Table 2. The predicted 24-h urinary Na excretion (PreUNa) equation was as follows:

$$
\begin{aligned}
\text { PreUNa }= & 5 \cdot 818 \times \mathrm{Na}_{\text {spot }}-25 \cdot 251 \times \mathrm{Cr}_{\text {spot }}+46 \cdot 951 \\
& \times \text { BMI }-53 \cdot 817 \times \text { Age }+4912 \cdot 3(\text { male }) \\
\text { PreUNa }= & 6 \cdot 998 \times \mathrm{Na}_{\text {spot }}-12 \cdot 814 \times \mathrm{K}_{\text {spot }}+34 \cdot 009 \\
& \times \text { Height }-56 \cdot 225 \times \text { Age }+1522 \cdot 75(\text { female })
\end{aligned}
$$

$R^{2}$ was 0.137 and 0.148 for male- and female-specific equations, respectively.

\section{Validity of four methods}

The four methods (INTERSALT ${ }^{(13)}$, Kawasaki ${ }^{(12)}$, Tanaka ${ }^{(11)}$ and PreUNa) are summarised in Table 1 . The performance of the four methods by comparing the laboratory-based method against the four methods is presented in Table 2 . In the new method, the mean estimated 24-h urinary $\mathrm{Na}$ excretion was higher in female compared with male $(P=0.09)$. Whereas, in the INTERSALT method and Kawasaki method, male had a higher estimate of 24-h urinary Na excretion $(P<0 \cdot 001)$. There was no significant difference of mean 24-h urinary Na excretion between the different gender group in the Tanaka method $(P=0.987)$.

The mean difference for estimating 24-h urinary $\mathrm{Na}$ excretion between the laboratory-based method and the INTERSALT method was $-0.07 \mathrm{~g} / \mathrm{d}$ (95\% CI $-0 \cdot 10$,
$0.25 \mathrm{~g} / \mathrm{d}$ ), while the difference between the laboratorybased method and the new method was $0.09 \mathrm{~g} / \mathrm{d}(95 \%$ CI $-0.22,0.04 \mathrm{~g} / \mathrm{d}$ ). Kawasaki and Tanaka methods highly overestimated the 24-h urinary Na excretion with the mean difference of $40.81 \mathrm{~g} / \mathrm{d}(95 \%$ CI $39.27,42.35 \mathrm{~g} / \mathrm{d})$ and $18.28 \mathrm{~g} / \mathrm{d}$ (95\% CI: $17.73,18.83 \mathrm{~g} / \mathrm{d}$ ), respectively. The intraclass correlation coefficient for PreUNa, INTERSALT, Kawasaki and Tanaka was 0.31 (95\% CI 0.22, 0.39), 0.10 (95\% CI $0.00,0.24), 0 \cdot 10$ (95\% CI $0.00,0.25)$ and 0.27 (95\% CI 0.12, 0.39), respectively.

Bland-Altman plots illustrating the bias of the four methods are shown in Fig. 2. The Bland-Altman plot of the PreUNa method showed a slightly negative association between the laboratory-measured value and the predicted value. There was a strong positive association between the laboratory-measured value and the predicated value from the Kawasaki and Tanaka methods. No significant association found for the INTERSALT method.

\section{Discussion}

This study developed a new method to estimate the 24-h urinary $\mathrm{Na}$ excretion using causal spot urine samples collected from an ongoing large-scale cluster randomised controlled trial. The study further evaluated the performances of the new method, INTERSALT method, Kawasaki method and the Tanaka method by comparing with laboratory-based method. The results showed that the Kawasaki method and the Tanaka method overestimated the 24-h urinary $\mathrm{Na}$ excretion and suggested that the INTERSALT method was the optimal choice to predict the 24-h urinary $\mathrm{Na}$ excretion in this study population. Despite there was a negative association found in the Bland-Altman plot of the new method, the new method had the potential to estimate $24-\mathrm{h} \mathrm{Na}$ excretion at the individual level.

The large mean differences for estimating the 24-h urinary $\mathrm{Na}$ excretion in the Kawasaki and Tanaka methods might be due to two primary reasons. Firstly, the Kawasaki and Tanaka methods were based on data from the Japanese population, while our research was based on northwestern Chinese population. The dietary pattern especially $\mathrm{Na}$ intake was different between the two populations with higher $\mathrm{Na}$ intake in the Chinese population. Secondly, the study participants were rural older hypertensive patients with an average less urinary volume $(1 \mathrm{l} / \mathrm{d})$, which led to a higher concentration of urinary $\mathrm{Na}$.

There were many studies in the literature to evaluate the best method to estimate the 24-h urinary Na excretion using spot urine with various findings ${ }^{(8-10,19-23)}$. A research conducted in general Brazilian adult population found that the Tanaka method underestimated the $\mathrm{Na}$ intake, while Kawasaki method overestimated the $\mathrm{Na}$ intake. The study concluded that these two methods can estimate salt consumption when individuals had an actual salt consumption 


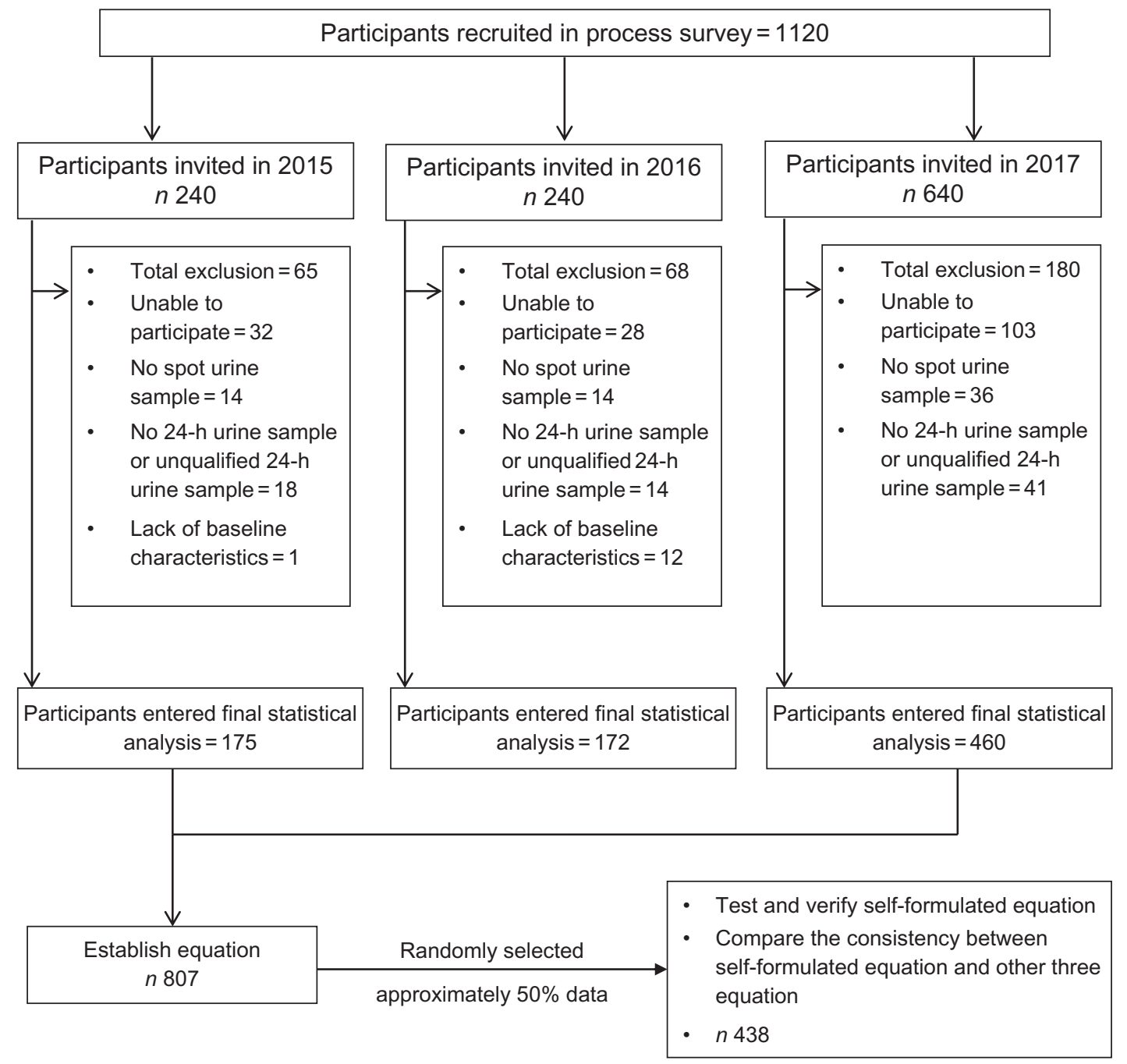

Fig. 1 Flow chart showed the details of the study. A total of 1120 participants were invited to participate in the survey from January 2015 to February 2017. A total of 807 individuals were included in the statistical analysis because of various reasons. The PreUNa equation was established by the data from all participants. The validity of the PreUNa equation was tested by the data from $50 \%$ randomly selected participants. PreUNa: predicted 24-h urinary $\mathrm{Na}$ excretion

Table 1 Four methods to estimate 24-h urinary sodium excretion

\begin{tabular}{|c|c|}
\hline Methods & Formula to predict $24-\mathrm{h}$ urinary $\mathrm{Na}$ excretion $(\mathrm{mg} / \mathrm{d})$ \\
\hline INTERSALT* & $\begin{array}{l}23 \times\left(\left(25.46+0.46 \times \mathrm{Na}_{\text {spot }}\right)-2.75 \times \mathrm{Cr}_{\text {spot }}-0.13 \times \mathrm{K}_{\text {spot }}+4.10 \times \mathrm{BMI}+0.26 \times \mathrm{Age}\right)(\text { male }) \\
23 \times\left(\left(5.07+0.34 \times \mathrm{Na}_{\text {spot }}\right)-2.16 \times \mathrm{Cr}_{\text {spot }}-0.09 \times \mathrm{K}_{\text {spot }}+2.39 \times \mathrm{BMl}+2.35 \times \mathrm{Age}^{2}-0.03 \times \mathrm{Age}^{2}\right)\left(\mathrm{female}^{2}\right)\end{array}$ \\
\hline Kawasaki* $^{*}$ & $\begin{array}{l}23 \times 16.3 \times\left(\mathrm{Na}_{\text {spot }} / \mathrm{Cr}_{\text {spot }} \times \mathrm{PreCr}\right)^{0.5} \\
\mathrm{PreCr}=15.12 \times \text { Weight }+7.39 \times \text { Height }-12.63 \times \text { Age }-79.9 \text { (male) } \\
\text { PreCr }=8.58 \times \text { Weight }+5.09 \times \text { Height }-4.72 \times \text { Age }-74.95 \text { (female) }\end{array}$ \\
\hline Tanaka* & $\begin{array}{l}23 \times 21.98 \times\left(\mathrm{Na}_{\text {spot }} / \mathrm{Cr}_{\text {spot }} \times \text { PreCr }\right)^{0.392} \\
\text { PreCr }=14.89 \times \text { Weight }+16.14 \times \text { Height }-2.04 \times \text { Age }-2244.45\end{array}$ \\
\hline PreUNa & $\begin{array}{l}5.818 \times \mathrm{Na}_{\text {spot }}-25.251 \times \mathrm{Cr}_{\text {spot }}+46.951 \times \mathrm{BMl}-53.817 \times \mathrm{Age}+4912.3(\text { male }) \\
6.998 \times \mathrm{Na}_{\text {spot }}-12.814 \times \mathrm{K}_{\text {spot }}+34.009 \times \text { Height }-56.225 \times \text { Age }+1522.75(\text { female })\end{array}$ \\
\hline
\end{tabular}

$\mathrm{Na}_{\text {spot }}$, spot urinary $\mathrm{Na} ; \mathrm{K}_{\text {spot }}$, spot urinary $\mathrm{K} ; \mathrm{Cr}_{\text {spot }}$, spot urinary creatinine; PreCr, predicted 24-h urinary creatinine; PreUNa, predicted 24-h urinary $\mathrm{Na}$ excretion; the units of concentration of $\mathrm{Na}_{\text {spot }}, \mathrm{K}_{\text {spot }}$ and $\mathrm{Cr}_{\text {spot }}$ are $\mathrm{mmol} / \mathrm{l}$; the unit of PreCr is $\mathrm{mg} / \mathrm{d}$. Weight and height are $\mathrm{kg}$ and $\mathrm{cm}$. The molecule weight of $\mathrm{Na}$ is $23 \mathrm{mg} / \mathrm{mmol}$.

${ }^{*}$ The equations are cited in reference 21.

close to the population mean value (9-12 $\mathrm{g} / \mathrm{d}$ for Tanaka and $12-18 \mathrm{~g} / \mathrm{d}$ for Kawasaki) ${ }^{(20)}$. Another research conducted in the Chinese adult showed the Kawasaki method has the best performance comparing with INTERSALT method and Tanaka method ${ }^{(21)}$. The INTERSALT method was considered as an effective alternative method to estimate 24-h urinary $\mathrm{Na}$ excretion in a research of Asian adults ${ }^{(17)}$. However, a study in Portugal showed that neither 
Table 2 Differences of four methods to estimate 24-h urinary sodium excretion $(\mathrm{g} / \mathrm{d})$

\begin{tabular}{|c|c|c|c|c|c|c|c|c|c|c|c|c|c|c|}
\hline & \multicolumn{2}{|c|}{ Measured } & \multicolumn{3}{|c|}{ PreUNa } & \multicolumn{3}{|c|}{ INTERSALT } & \multicolumn{3}{|c|}{ Kawasaki } & \multicolumn{3}{|c|}{ Tanaka } \\
\hline & Mean & SD & Mean & SD & & Mean & SD & & Mean & & SD & Mean & & SD \\
\hline \multicolumn{15}{|l|}{ Mean } \\
\hline All & 3.03 & 1.52 & $3 \cdot 12$ & & 0.66 & 2.96 & & $1 \cdot 13$ & 43.85 & & $16 \cdot 71$ & 21.32 & & $6 \cdot 21$ \\
\hline Male & $2 \cdot 67$ & 1.34 & $2 \cdot 82$ & & 0.52 & 3.76 & & 0.83 & 47.00 & & $19 \cdot 11$ & $21 \cdot 18$ & & $6 \cdot 78$ \\
\hline Female & 3.41 & 1.61 & 3.43 & & 0.64 & $2 \cdot 15$ & & 0.74 & 40.64 & & 13.15 & 21.46 & & 5.58 \\
\hline $\begin{array}{l}\text { Mean } \\
\quad \text { difference }\end{array}$ & Reference & & 0.09 & & & -0.07 & & & $40 \cdot 81$ & & & $18 \cdot 28$ & & \\
\hline $95 \% \mathrm{Cl}$ & & & & $-0.22,0.04$ & & & $-0.10,0.25$ & & & $39.27,42 \cdot 35$ & & & $17 \cdot 7,18 \cdot 83$ & \\
\hline $\begin{array}{l}\text { ICC } \\
\quad 95 \% \mathrm{Cl}\end{array}$ & Reference & & 0.31 & $0.22,0.39$ & & 0.10 & $0.00,0.24$ & & $0 \cdot 10$ & $0.000,0.25$ & & 0.27 & $0.12,0.39$ & \\
\hline
\end{tabular}

ICC, intraclass correlation coefficient.

(a)

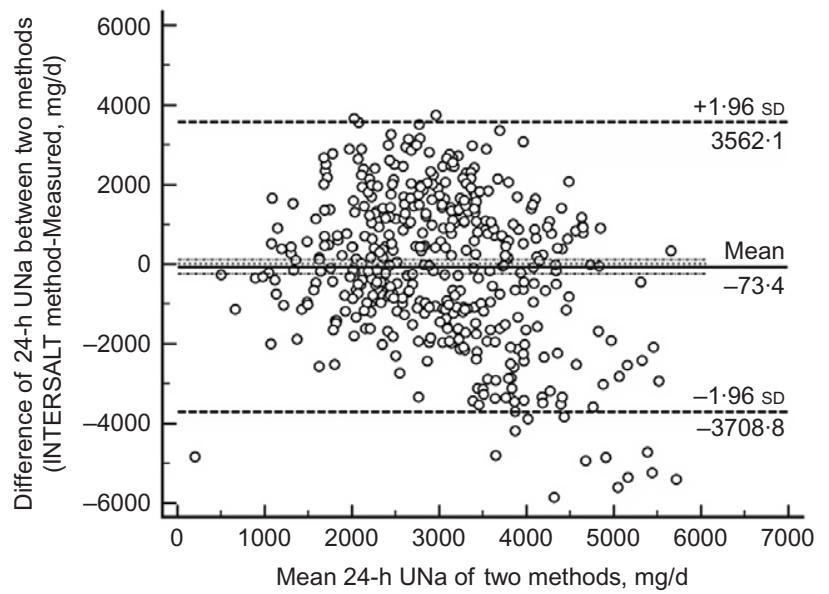

(c)

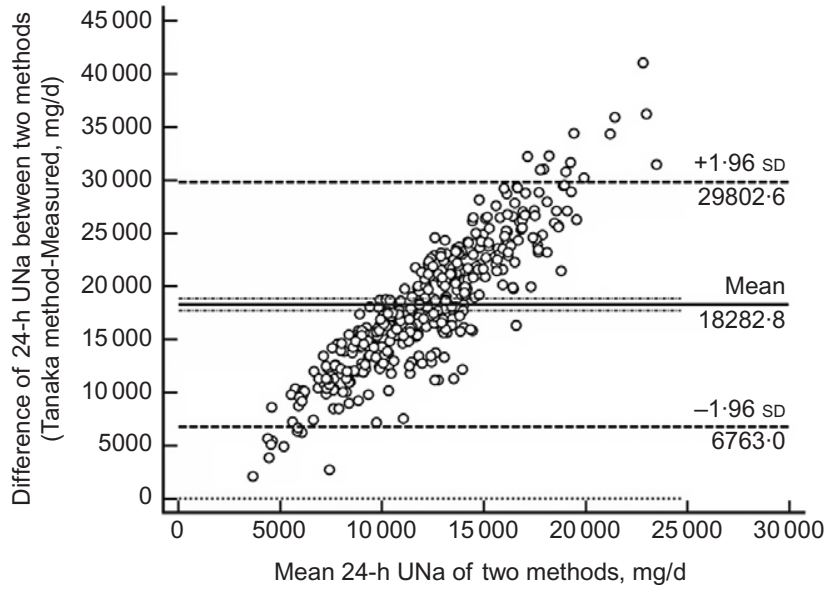

(b)

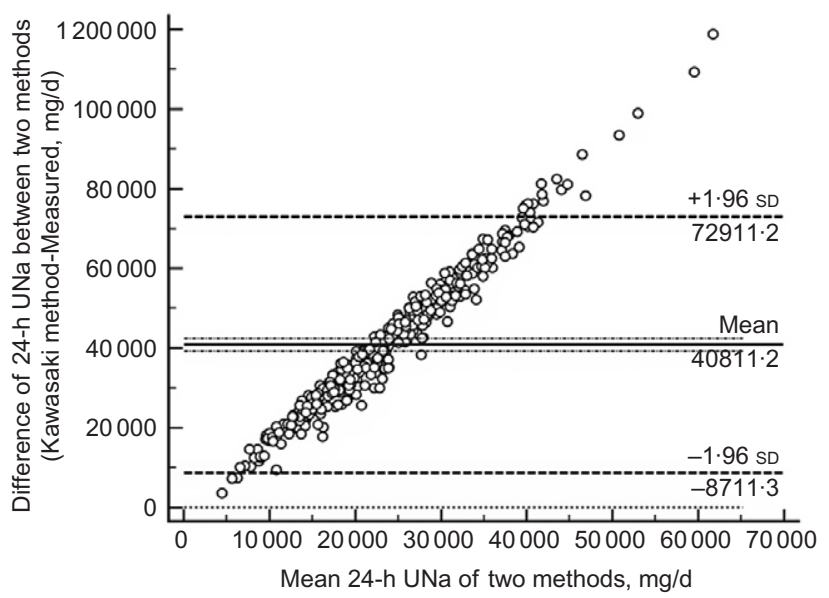

(d)

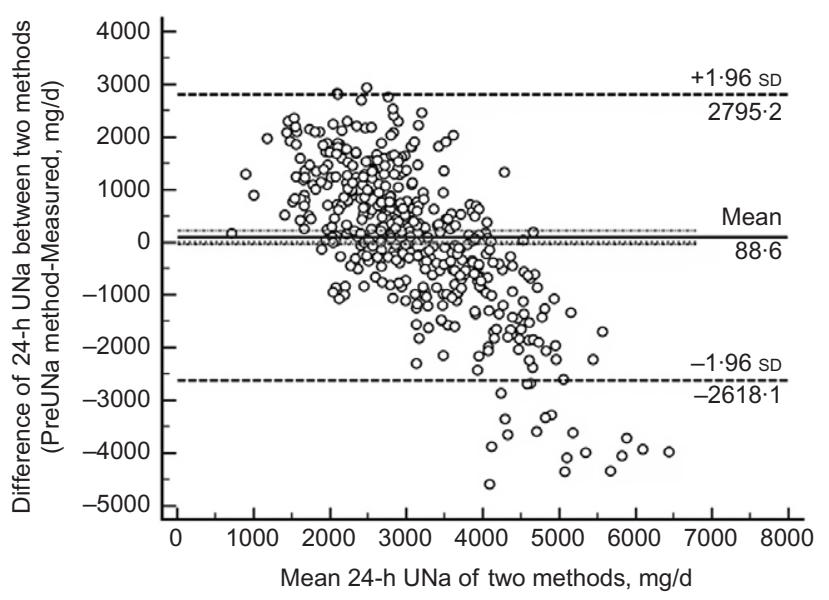

Fig. 2 Bland-Altman plots showed the mean difference between measured and predicted 24-h urinary Na excretion of INTERSALT (a), Kawasaki (b), Tanaka (c) and PreUNa (d) methods. The long solid line represents the mean bias. The two short dotted lines represent the $95 \% \mathrm{Cl}$ of the mean bias. The two long dotted lines represent the upper/lower limits of agreement $( \pm 1.96 \mathrm{SD})$. PreUNa: predicted 24-h urinary $\mathrm{Na}$ excretion

of the methods (INTERSALT, Kawasaki, Tanaka and NHANES) were able to actually estimate the 24-h urinary $\mathrm{Na}$ and $\mathrm{K}$ excretion ${ }^{(22)}$. Another research involved young Black and other adults evaluated validity of predictive equations named Brown for 24-h urine Na excretion. The Brown method was gender-specific equations by using spot urine
$\mathrm{Na}, \mathrm{K}$, creatinine concentrations, age and BMI. The results showed that mean biases from Brown method were not significant ( -167 to $122 \mathrm{mg}$ ) among Blacks, while it was greatest on other adults $(-247 \mathrm{mg}, 95 \% \mathrm{CI}-348,-151 \mathrm{mg})$ when using overnight spot urine samples ${ }^{(23)}$. Those inconsistent findings indicated that ethnicity and the dietary pattern 
may be important confounding factors to predict the urinary $\mathrm{Na}$ excretion.

In addition, 24-h urinary $\mathrm{Na}$ excretion prediction was dependent on the type of the urine sample. A systematic review study compared spot, timed and overnight urine samples in estimating 24-h Na excretion from forty-three studies published from 1979 to 2010. The correlation coefficient ranged from 0.17 to 0.94 , and there was no recommendation provided $^{(24)}$. The predication of the 24-h urinary $\mathrm{Na}$ excretion was also dependent on the time of the spot urine samples. The INTERSALT method had the best prediction in the afternoon specimens ( $-90 \mathrm{mg}$; 95\% CI $-208,28 \mathrm{mg}$ ), while Tanaka had the best performance in the overnight specimens ${ }^{(23)}$. In a Chinese population study, the second morning urine samples and the post-meridiem samples were used to estimate the 24-h urine samples among 222 hypertensive patients. The study found that Kawasaki method was useful for estimating 24-h Na excretion mean levels from second morning urine samples but not reliable for estimating individual $\mathrm{Na}$ excretion. A recent research compared different published equations for predicting 24-h urinary $\mathrm{K}$ excretion and its accuracy varies by spot urine collection time, age, sex and race ${ }^{(25)}$. There is however currently no reliable method across all sex, race and age groups.

There were several limitations of this study. Firstly, the findings of this study were only limited to one province of the Salt Substitute and Stroke Study trial. There were five provinces involved in the Salt Substitute and Stroke Study trial. The findings to apply to the other provinces remained unknown. Secondly, there was only one spot urine sample collected in this study. Previous research found that a single spot urine sample was not a valid indicator to estimate individual level $\mathrm{Na}$ intake ${ }^{(26)}$. Thirdly, this study was limited to its sample size and study population. A large-scale study involving a heterogeneous population is needed in the future research.

\section{Conclusion}

Compared with laboratory-measured 24-h urinary Na excretion, the predicted outcomes of the INTERSALT method and the new method showed no statistically significant bias. These two methods had the potential to estimate individual level 24-h urinary $\mathrm{Na}$ excretion in this study population. The extrapolation of the results needs to be careful. Future research is required to establish a more reliable method to estimate 24-h urinary Na excretion.

\section{Acknowledgements}

Acknowledgements: The authors thank all the participants and investigators involved in this study. The authors also thank the central laboratory in Beijing. Financial support:
This research is a sub-study of the Salt Substitute and Stroke Study (SSaSS), which is supported by the National Health and Medical Research Council of Australia (NHMRC) Project Grant (APP1049417), NHMRC Program Grant (APP1052555), NHMRC Centre for Research Excellence Grant (APP1117300) and Top Discipline of Public Health and Prevent Medicine (NXYLXK2017A08), Education Department of Ningxia, China. Conflict of interest: None. Authorship: Y.Z., M.T. and Y.Z. participated in the study and critical review of the manuscript. Y.Z. and W.L. drafted the manuscript. W.L. conducted the statistical analysis. Y.Z. and Y.Z. oversaw the implementation of the study. S.L., X.L., X.C., X.L., T.Y., T.Z. and F.W. collected the data and specimen collection. All authors read and approved the final manuscript. Ethics of human subject participation: This study was conducted according to the guidelines laid down in the Declaration of Helsinki and all procedures involving research study participants were approved by the Medical Ethics Review Committee of Ningxia Medical University, The Institutional Review Board of Peking University Health Science Center and The Ethics Committee of University of Sydney. Written informed consent was obtained from all patients.

\section{Supplementary material}

For supplementary material accompanying this paper visit https://doi.org/10.1017/S1368980019005019

\section{References}

1. Erdem Y, Arici M, Altun B et al. (2010) The relationship between hypertension and salt intake in Turkish population: SALTURK study. Blood Press 19, 313-318.

2. Ha SK (2014) Dietary salt intake and hypertension. Electrolyte Blood Press 12, 7-18.

3. Neal B (2014) Dietary salt is a public health hazard that requires vigorous attack. Can J Cardiol 30, 502-506.

4. D'Elia L, Galletti F \& Strazzullo P (2014) Dietary salt intake and risk of gastric cancer. Cancer Treat Res 159, 83-95.

5. World Health Organization (2012) Guideline: Sodium Intake for Adults and Children. Geneva, Switzerland: World Health Organization.

6. Mclean RM (2014) Measuring population sodium intake: a review of methods. Nutrients $\mathbf{6}, 4651-4662$.

7. World Health Organization (2011) Strategies to Monitor and Evaluate Population Sodium Consumption and Sources of Sodium in the Diet: Report of a Joint Technical Meeting Convened by $\mathrm{WHO}$ and the Government of Canada. Geneva: WHO.

8. Mann SJ \& Gerber LM (2010) Estimation of 24-hour sodium excretion from spot urine samples. J Clin Hypertens 12, 174.

9. Ortega RM, Lopez-Sobaler AM, Ballesteros JM et al. (2011) Estimation of salt intake by $24 \mathrm{~h}$ urinary sodium excretion in a representative sample of Spanish adults. Br J Nutr 105, 787-794

10. Toft U, Cerqueira C, Andreasen AH et al. (2013) Estimating salt intake in a Caucasian population: can spot urine substitute 24-hour urine samples? Eur J Prev Cardiol 21, 1300-1307. 
11. Tanaka T, Okamura T, Miura K et al. (2002) A simple method to estimate populational 24-h urinary sodium and potassium excretion using a casual urine specimen. J Hum Hypertens 16, 97-103

12. Kawasaki T IK, Uezono K \& Sasaki H (1993) A simple method for estimating $24 \mathrm{~h}$ urinary sodium and potassium excretion from second morning voiding urine specimen in adults. Clin Exp Pharmacol Physiol 20, 7.

13. Brown IJ, Dyer AR, Chan Q et al. (2013) Estimating 24-hour urinary sodium excretion from casual urinary sodium concentrations in Western populations: the INTERSALT study. Am J Epidemiol 177, 1180-1192.

14. Zhang T, Chang X, Liu W et al. (2017) Comparison of sodium, potassium, calcium, magnesium, zinc, copper and iron concentrations of elements in 24-h urine and spot urine in hypertensive patients with healthy renal function. J Trace Elem Med Biol 44, 104-108.

15. Bruce Neal MT, Li N, Elliott P et al. (2017) Rationale, design, and baseline characteristics of the Salt Substitute and Stroke Study (SSaSS) - a large-scale cluster randomized controlled trial. Am Heart J 188, 109-107.

16. WHO \& PAHO (2010) Protocol for Population Level Sodium Determination in 24-hour Urine Samples. http://www.paho. org/hq/index.php?option=com_docman\&task=doc_view\& gid=21488\&Itemid= $($ accessed October 2018) .

17. Whitton C, Gay GM, Lim RB et al. (2016) Evaluation of equations for predicting 24-hour urinary sodium excretion from casual urine samples in Asian adults. J Nutr 146, $1609-1615$.

18. Bland JM \& Altman DG (1986) Statistical methods for assessing agreement between two methods of clinical measurement. Lancet 1, 931-936.
19. Kawasaki T, Ueno M, Uezono K et al. (1982) Average urinary excretion of sodium in 24 hours can be estimated from a spot-urine specimen. Jpn Circ J 46, 948-953.

20. Mill JG, Rodrigues SL, Baldo MP et al. (2015) Validation study of the Tanaka and Kawasaki equations to estimate the daily sodium excretion by a spot urine sample. Rev Bras Epidemiol 18, Suppl. 2, 224-237.

21. Peng Y, Li W, Wang Y et al. (2016) Validation and assessment of three methods to estimate 24-h urinary sodium excretion from spot urine samples in Chinese adults. PLOS ONE 11, e0149655.

22. Polonia J, Lobo MF, Martins L et al. (2016) Estimation of populational 24-h urinary sodium and potassium excretion from spot urine samples: evaluation of four formulas in a large national representative population. J Hypertens 34, 1.

23. Cogswell M, Wang CY, Chen TC et al. (2013) Validity of predictive equations for 24-h urinary sodium excretion in adults aged 18-39 y. Am J Clin Nutr 98, 1502-1513.

24. Ji C, Sykes L, Paul C et al. (2012) Systematic review of studies comparing 24-hour and spot urine collections for estimating population salt intake. Rev Panam Salud Publica 32, 307-315.

25. Mercado CI, Cogswell ME, Loria CM et al. (2018) Validity of predictive equations for 24-h urinary potassium excretion based on timing of spot urine collection among adults: the MESA and CARDIA Urinary Sodium Study and NHANES Urinary Sodium Calibration Study. Am J Clin Nutr 108, 532-547.

26. Allen NB, Zhao L, Loria CM et al. (2017) The validity of predictive equations to estimate 24-hour sodium excretion: the MESA and CARDIA urinary sodium study. Am J Epidemiol 186, 149. 